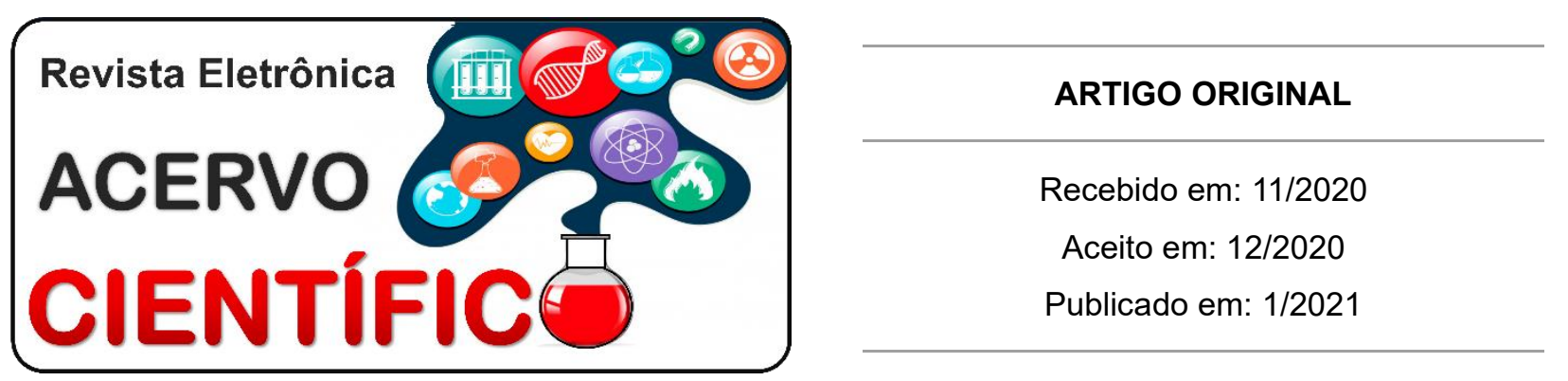

\title{
Endometriose e a qualidade de vida das mulheres acometidas
}

\author{
Endometriosis and quality of life of the women affected
}

\section{Endometriosis e calidad de vida de las mujeres afectadas}

Beatriz Valente Baetas ${ }^{1 \S}$, Bianca Valezin Bretas ${ }^{1 \S}$, Caroline Molina Maziviero ${ }^{1}$, Geovanna Zucareli de Moraes ${ }^{1 \S}$, Larissa Tocci Savi Rodrigues ${ }^{1 \S}$, Alan Zanluchi², Wagner Alves de Souza Júdice ${ }^{3 *}$.

\begin{abstract}
Resumo: Trata-se de um estudo transversal e qualitativo no qual avaliamos a qualidade de vida de 640 mulheres acometidas de endometriose por meio da aplicação do questionário EHP-30, pois a doença causa significativos impactos psicológicos em âmbito emocional decorrentes de diversos fatores como dores crônicas, infertilidade, redução das atividades, isolamento social, impacto econômico, interferência nas relações afetivas e familiares, dentre outros que prejudicam a qualidade de vida dessas mulheres. Verificamos que $66,43 \%$ apresentaram baixa qualidade de vida. A Dimensão dor afetou $78,04 \%$ das participantes. Idades entre 14-20 e 41-53 anos foram as mais acometidas pela dor. A Seção Filhos foi pouco impactada afetando apenas 22,99\% das mulheres. A seção Gravidez apresentou maior relevância na qualidade de vida caracterizada pela infertilidade atingindo $55,34 \%$ das mulheres. A seção Tratamento, o segundo fator mais impactante, atingiu $40,39 \%$ das participantes. Dentre as mulheres $32,26 \%$ relataram evitar relações sexuais por apresentarem dispareunia e 40,39\% estavam frustradas com o próprio tratamento. Conclui-se que a dor decorrente da endometriose afeta a vida social da mulher, altera seu interesse sexual, modifica sua concepção de mulher devido à infertilidade, ocasiona alterações de humor, depressão e irritabilidade reduzindo sua qualidade de vida.
\end{abstract}

Palavras-chave: Endometriose, Qualidade de vida, Dismenorrei.

\begin{abstract}
This is a cross-sectional and qualitative study in which we assessed the quality of life of 640 women with endometriosis through the application of the EHP-30 questionnaire, as the disease causes significant psychological impacts on an emotional level due to several factors such as chronic pain, infertility, reduced activities, social isolation, economic impact, interference in affective and family relationships, among others that affect the quality of life of these women. We found that $66.43 \%$ had a low quality of life. The Pain dimension affected $78.04 \%$ of the participants. Women with ages between $14-20$ and $41-53$ years were the most affected by pain. The Children section was little impacted, affecting only $22.99 \%$ of women. The Pregnancy section showed greater relevance in the quality of life characterized by infertility, reaching $55.34 \%$ of women. The Treatment section, the second most impacting factor, reached $40.39 \%$ of the participants. Among women, $32.26 \%$ reported avoiding sexual intercourse due to dyspareunia and $40.39 \%$ were frustrated with their own

1 Universidade de Mogi das Cruzes (UMC), Mogi das Cruzes - SP.

2Santa Casa de Misericórdia de Mogi das Cruzes, Mogi das Cruzes - SP.

${ }^{3}$ Centro Interdisciplinar de Investigação Bioquímica, Universidade de Mogi das Cruzes (UMC), Mogi das Cruzes - SP. *E-mail: wagnerjudice@gmail.com

$\S$ As autoras trabalharam igualmente para o desenvolvimento desse estudo.
\end{abstract}


treatment. It is concluded that the pain resulted from endometriosis affects the social life of women, changes their sexual interest, changes their conception of women due to infertility, causes mood changes, depression and irritability reducing their quality of life.

Keywords: Endometriosis, Quality of life, Dysmenorrhea.

Resumen: Se trata de un estudio transversal y cualitativo en el que evaluamos la calidad de vida de 640 mujeres con endometriosis mediante la aplicación del cuestionario EHP-30, ya que la enfermedad provoca importantes impactos psicológicos a nivel emocional debido a varios factores como el dolor crónico, infertilidad, reducción de actividades, aislamiento social, impacto económico, injerencia en las relaciones afectivas y familiares, entre otros que afectan la calidad de vida de estas mujeres. Encontramos que el $66,43 \%$ tenía una baja calidad de vida. La dimensión del dolor afectó al 78,04\% de los participantes. Las edades entre 14-20 y 41-53 años fueron las más afectadas por el dolor. La sección de Niños se vio poco afectada, afectando solo al 22,99\% de las mujeres. El apartado de Embarazo mostró una mayor relevancia en la calidad de vida debido a la infertilidad, afectando al $55,34 \%$ de las mujeres. La sección de Tratamiento, el segundo mayor factor, llegó al 40,39\% de las participantes. Entre las mujeres, el 32,26\% informó haber evitado tener relaciones sexuales porque tenían dispareunia y el $40,39 \%$ sesintió frustrada con su propio tratamiento. Se concluye que el dolor derivado de la endometriosis afecta la vida social de la mujer, cambia su interés sexual, modifica su concepción de mujer por infertilidad y provoca cambios de humor, depresión e irritabilidad reduciendo su calidad de vida.

Palabras clave: Endometriosis, Calidad de vida, Dismenorrea.

\section{INTRODUÇÃO}

A endometriose (EDM) é caracterizada, pela presença de tecido endometrial ectópico, que afeta principalmente as mulheres na idade reprodutiva, é uma doença crônica, conhecida por acarretar sintomas desconfortantes e substancialmente prejudiciais para a qualidade de vida das mulheres. A incidência da endometriose varia de $2-15 \%$ nas mulheres em idade reprodutiva, no entanto no grupo de mulheres inférteis a incidência de endometriose é de $20-50 \%$, e no grupo de mulheres com dor pélvica crônica de $30-80 \%$ (MINSON FP, et al., 2012; SOLIMAN AM, et al., 2016; BUCK LOUIS GM, et al., 2011).

O quadro clínico das pacientes com endometriose é variado, 3 a $22 \%$ delas são assintomáticas. Porém, a grande maioria apresenta como sintomas físicos mais comuns a dismenorreia, dispareunia, dor pélvica crônica (DPC), disúria, disquezia e infertilidade. Por se tratar de uma doença crônica e dolorosa, ela exibe importância por apresentar impacto significativo na qualidade de vida das mulheres acometidas (DE MARQUI ABT, 2014).

A queda da qualidade de vida das pacientes, entretanto, não ocorre apenas do âmbito físico e de suas morbidades advindas dos sintomas que a EDM acarreta, a doença causa significativos impactos psicológicos em âmbito emocional decorrentes de diversos fatores como, por exemplo, as dores crônicas, infertilidade, redução das atividades, isolamento social, impacto econômico, interferência nas relações afetivas e familiares, dentre outros. Dessa forma, todos estes fatores devem ser levados em conta no diagnóstico clínico e tratamento (MINSON FP, et al., 2012).

Além disso, sintomas psicológicos como dificuldades nas relações interpessoais afetivas e na sexualidade, estresse, depressão e ansiedade são relatados como aqueles que mais prejudicam a vida das mulheres com endometriose. A depressão é uma característica clínica que sempre deve ser levada em conta e os níveis são maiores ainda em mulheres acometidas pela endometriose profunda e pessoas que apresentam algum tipo de doença. Portanto, deve-se pensar em intervenções com estratégias que auxiliem na luta contra a depressão e o estresse dessas pacientes (DONATTI L, et al., 2017). A infertilidade também deve ser levada em conta no diagnóstico e tratamento. Mulheres que apresentam a doença possuem uma chance vinte vezes maior de se tornarem inférteis (LUIZ RP, et al., 2005). 
Em relação ao diagnóstico clínico, analisam-se as manifestações da paciente com suspeita de endometriose, sendo que, os sintomas são mais intensos durante o período pré-menstrual e durante a menstruação, podendo evoluir para uma dor incapacitante (HERNÁNDEZ-VALENCIA M, 2009; CARDOSO EPS, et al., 2011).

O diagnóstico padrão ouro é a realização de videolaparoscopia com biopsia das lesões para análise anatomopatológca. Além deste, a Ultrassonografia transvaginal especializada com preparo retal pode identificar a forma retrocervical ou septo retrovaginal da doença. Assim, a USG constitui um método eficaz para o diagnóstico de focos de células endometriais em estágios iniciais (CARDOSO EPS, et al., 2011; NACUL AP e SPRITZER PM, 2010).

Entretanto, a Ressonância Magnética Nuclear mostra-se sofisticada no diagnóstico da endometriose profunda, por detectar massa pélvica, mesmo que não ultrapasse a qualidade do USG. Não existe um marcador sérico específico destinado apenas à endometriose. Dessa forma, para um diagnóstico eficaz é necessário a correlação entre os exames de videolaparoscopia, ultrassonografia e análise dos sintomas sentidos pela mulher, somados a exame físicos e ginecológicos (CARDOSO EPS, et al., 2011).

O objetivo deste estudo foi analisar os efeitos da endometriose na qualidade de vida de mulheres acometidas e identificar o impacto em âmbito físico, psíquico e social mediante aplicação do questionário EHP-30.

\section{MÉTODOS}

Trata-se de um estudo qualitativo, descritivo e de recorte transversal, por meio da aplicação, utilizando Google Forms, do questionário EHP-30 o qual é dividido em questionário central (17 questões) e questionário modular (21 perguntas). Avaliou-se o impacto da doença nos seis domínios: trabalho (Seção A), relacionamento com crianças (Seção $B$ ), relações sexuais (Seção $C$ ), relacionamento com médico (Seção $D$ ), tratamento (Seção E) e infertilidade-gravidez (seção F).

Foram avaliadas 640 portadoras de endometriose. Os dados foram tabulados em programa Microsoft Excel 365 e analisados estatisticamente em programa Statistical Package for Social Sciencies (SPSS) versão 18.0 e nível de significância $p=0,05$ e intervalo de confiança de 95\% (MENGARDA CV, et al., 2019).

Utilizou-se a escala de Likert para pontuação das respostas. Para as variáveis quantitativas calculou-se a média, como medida de tendência central. Para análise de diferença estatística entre domínios foi realizado o t-test. O questionário foi aplicado em grupos fechados de mulheres com endometriose da plataforma Facebook cujos membros eram previamente checadas pelas moderadoras. Foram incluídas mulheres portadoras de endometriose sintomáticas e assintomáticas independentemente de estarem ou não em tratamento. O estudo seguiu os princípios éticos contidos na declaração de Helsinque da Associação Médica Mundial. O projeto foi submetido ao Comitê de Ética da Universidade de Mogi das Cruzes (UMC), sobre o número CAAE 02649118.1.0000.5497 e aprovado sobre o parecer consubstanciado 3.059.276 de acordo com os termos da Resolução CNS no 466/12.

\section{RESULTADOS}

O estudo avaliou 640 mulheres com média de idade de 32,52 anos (mínima de 14 e máxima de 53 anos). $\mathrm{Na}$ avaliação da frequência de impactos na qualidade de vida por meio do questionário central, verificamos que $41,31 \%$ das respostas foram "muitas vezes" com frequência de 7,02 implicando, baixa qualidade de vida das mulheres que participaram do estudo (Tabela 1).

Em outra análise, as participantes foram divididas em dois grupos: com impacto na qualidade de vida devido as respostas "sempre (ESCORE 4) e muitas vezes (ESCORE 3)", correspondendo a 66,43\% das participantes; e sem impacto totalizando $33,57 \%$. Verificamos que a maioria de nossa amostragem apresentou sua qualidade de vida impactada pela endometriose (Tabela 1).

Abordando o Questionário Central a Dimensão Dor para o termo muitas vezes apresentou escore de 10,7484, sendo o maior escore entre todas as dimensões e termos, significando que a dor é um parâmetro 
que impacta muito a qualidade de vida das mulheres com endometriose. Para as demais Dimensões Controle e impotência, Bem-estar emocional, Suporte social, Autoimagem, os maiores escores ficaram para os termos sempre, muitas vezes, sempre e muitas vezes, respectivamente. Isso significa que todas as dimensões impactam negativamente a qualidade de vida dessas mulheres (Tabela 2).

Tabela 1 - Frequência de impacto na qualidade de vida e escores EHP-30 para o questionário central em mulheres com endometriose.

\begin{tabular}{ccccccc}
\hline \multicolumn{7}{c}{ Frequência de impacto } \\
\hline Escore & $\mathbf{0}$ & $\mathbf{1}$ & $\mathbf{2}$ & $\mathbf{3}$ & $\mathbf{4}$ & Total \\
\hline $\mathbf{N}$ & 565 & 748 & 2341 & 4497 & 2734 & 10885 \\
\hline Frequência & 0,88 & 1,17 & 3,66 & 7,02 & 4,27 & - \\
\hline$\% \mathbf{N}$ & $5,19 \%$ & $6,87 \%$ & $21,51 \%$ & $41,31 \%$ & $25,12 \%$ & $100 \%$ \\
\hline
\end{tabular}

Legenda: $\mathrm{N} \rightarrow$ corresponde à somatória de respostas para todas as questões do Questionário Central em um único escore. Frequência $\rightarrow$ relação entre o número de resposta em um escore pelo número de participantes. Número de participantes $\rightarrow$ $\mathrm{n}=640.0$ nunca, $1=$ raramente, $2=$ às vezes, $3=$ muitas vezes, $4=$ sempre.

Fonte: Baetas BV, et al., 2020.

Quando se considera a média dos escores para cada Dimensão na população em estudo, verifica-se que a Dimensão Controle e impotência (escore 3,069) foi a que mais impactou negativamente a qualidade de vida das participantes seguida da Dimensão Bem-estar emocional $(2,869)$ com valor de $p=0,02$. Dessa forma, essa mulher tem sua qualidade de vida mais impactada pela falta do controle e sua impotência sobre a endometriose do que as demais dimensões que abrangem o questionário central. Além disso, verifica-se que a autoimagem foi a que menos impactou a qualidade de vida sendo estatisticamente diferentes das demais dimensões $(p=0,0455)$ (Tabela 2).

Tabela 2 - Distribuição e média dos escores de acordo com a Dimensão e termo baseado no questionário central das participantes do estudo.

\begin{tabular}{ccccccc}
\hline Dimensões & $\mathbf{0}$ & $\mathbf{1}$ & $\mathbf{2}$ & $\mathbf{3}$ & $\mathbf{4}$ & Média dos escores \\
\hline Dor & 0 & 0,6376 & 3,9594 & 10,7484 & 5,5875 & 2,614 \\
Controle e impotência & 0 & 0,0906 & 0,5344 & 2,1891 & 3,3250 & 3,069 \\
Bem estar emocional & 0 & 0,1422 & 1,2094 & 3,7406 & 3,5125 & 2,869 \\
Suporte social & 0 & 0,2156 & 1,1187 & 3,2344 & 3,8750 & 2,811 \\
Autoimagem & 0 & 0,0828 & 0,4938 & 1,1672 & 0,7875 & 2,531 \\
\hline
\end{tabular}

Fonte: Baetas BV, et al., 2020.

Analisando a dimensão Dor no Questionário Central verifica-se que $44,64 \%$ das respostas se concentraram no termo muitas vezes e $16,65 \%$ em sempre, ou seja, $62,29 \%$ das respostas indicavam que as mulheres apresentavam qualidade de vida impactada negativamente pela dor. Tal fato se reforça com uma porcentagem de distribuição de respostas, relativa à dimensão dor, de apenas 5,06\% em nunca e de 8,09\% em raramente.

Tabela 3 - Distribuição dos escores de acordo com a Seção e termo baseado no questionário modular das participantes do estudo.

\begin{tabular}{cccccc}
\hline Seção & $\mathbf{0}$ & $\mathbf{1}$ & $\mathbf{2}$ & $\mathbf{3}$ & $\mathbf{4}$ \\
\hline Trabalho & 0 & 0,3906 & 0,5187 & 0,4922 & 1,056 \\
Filhos & 0 & 0,2859 & 0,09687 & 0,1734 & 0,4125 \\
Sexo & 0 & 0,2781 & 0,2281 & 0,1922 & 1,2375 \\
Médico & 0 & 0,6547 & 0,3656 & 0,4031 & 2,2687 \\
Tratamento & 0 & 0,5344 & 0,2500 & 0,3797 & 2,3687 \\
Gravidez & 0 & 0,3562 & 0,3468 & 0,2765 & 3,3562 \\
\hline
\end{tabular}

Legenda: $0=$ nunca, $1=$ raramente, 2 = às vezes, $3=$ muitas vezes, 4

= sempre. Fonte: Baetas BV, et al., 2020 . 
Ainda em relação à dimensão Dor, observou-se que as faixas etárias de 14 a 20 anos e 41 a 53 anos apresentaram maiores médias de escores e, portanto, sofriam maior impacto na qualidade de vida, contudo foi observada diferença estatística somente para a faixa de 14 a 20 (valor de $p=0,0342$ ) em relação às demais faixas etárias.

A Seção Filhos do questionário modular apresentou para o termo sempre um escore de $0,4125(p=0,0171)$ e Gravidez com 3,3562 ( $p=0,0072$ ). Portanto, com exceção da seção Filhos (pouco afetada), todas as demais seções são fortemente impactadas negativamente, sendo a Gravidez a de maior relevância. Ou seja, a dificuldade de engravidar e ou manter a gravidez são fatores que muito afetam a qualidade de vida de uma mulher com endometriose (Tabela 3).

Infertilidade (Seção F) apresentou o maior impacto sobre a qualidade de vida das mulheres do estudo com média de escore de 2,576 ( $p=0,0011$ ), sendo estatisticamente diferentes das demais seções corroborando 0 observado pontualmente no termo sempre. Relacionamento com crianças-Filhos (Seção B) apresentou a menor média sendo estatisticamente diferente das demais seções ( $p=0,0228)$ (Tabela 4$)$.

Tabela 4 - Avaliação dos escores por questão e por seção com base no Questionário Modular.

\begin{tabular}{|c|c|c|c|c|c|c|c|c|c|c|}
\hline \multirow[b]{2}{*}{ Seção ${ }^{a}$} & \multirow[b]{2}{*}{ Questão } & \multicolumn{5}{|c|}{ Número de respostas ${ }^{b}$} & \multirow{2}{*}{$\begin{array}{l}\text { Soma dos } \\
\text { escores }^{c}\end{array}$} & \multirow{2}{*}{$\begin{array}{l}\text { Número de } \\
\text { respostas }\end{array}$} & \multirow{2}{*}{$\begin{array}{c}\text { Média } \\
\text { escore por } \\
\text { questão }\end{array}$} & \multirow{2}{*}{$\begin{array}{c}\% \\
\text { Afetadas }^{d}\end{array}$} \\
\hline & & 0 & 1 & 2 & 3 & 4 & & & & \\
\hline \multirow{2}{*}{ A } & Q19 & 164 & 134 & 62 & 66 & 41 & 620 & 467 & 1,328 & \multirow{2}{*}{1,675} \\
\hline & Q20 & 84 & 116 & 104 & 39 & 128 & 953 & 471 & 2,023 & \\
\hline \multirow{2}{*}{ B } & Q22 & 69 & 92 & 16 & 18 & 29 & 294 & 224 & 1,312 & \multirow{2}{*}{1,384} \\
\hline & Q23 & 62 & 91 & 15 & 19 & 37 & 326 & 224 & 1,455 & \\
\hline \multirow{3}{*}{ C } & Q25 & 149 & 212 & 24 & 47 & 187 & 1149 & 619 & 1,856 & \multirow{3}{*}{1,730} \\
\hline & Q26 & 170 & 279 & 44 & 46 & 80 & 825 & 619 & 1,333 & \\
\hline & Q27 & 129 & 178 & 73 & 41 & 198 & 1239 & 619 & 2,002 & \\
\hline \multirow{2}{*}{ D } & Q28 & 164 & 213 & 82 & 57 & 114 & 1004 & 630 & 1,594 & \multirow{2}{*}{1,865} \\
\hline & Q29 & 117 & 206 & 35 & 29 & 249 & 1359 & 636 & 2,137 & \\
\hline \multirow{2}{*}{ E } & Q34 & 137 & 171 & 45 & 47 & 168 & 1074 & 568 & 1,891 & \multirow{2}{*}{1,985} \\
\hline & Q35 & 120 & 171 & 35 & 34 & 211 & 1187 & 571 & 2,079 & \\
\hline \multirow{2}{*}{$\mathbf{F}$} & Q37 & 71 & 113 & 57 & 28 & 269 & 1387 & 538 & 2,578 & \multirow{2}{*}{2,576} \\
\hline & Q38 & 71 & 115 & 54 & 31 & 268 & 1388 & 539 & 2,575 & \\
\hline
\end{tabular}

Legenda: a) Trabalho (Seção A); Relacionamento com crianças-Filhos (Seção B); Relações sexuais-Sexo (Seção C); Relacionamento com médico (Seção D); Tratamento (Seção E); Infertilidade-Gravidez (seção F). b) Nunca $\rightarrow 0$; Raramente $\rightarrow 1$; Às vezes $\rightarrow 2$; Muitas vezes $\rightarrow 3$; Sempre à 4. c) multiplicação do número de resposta em cada termo pelo seu valor de acordo com a escala de Likert. d) Porcentagem de mulheres que reportaram serem afetadas em cada seção do questionário modular sendo considerados as que responderam os termos muitas vezes e sempre. Fonte: Baetas BV, et al., 2020.

\section{DISCUSSÃO}

O presente estudo retrata diversos aspectos relacionados à qualidade de vida de mulheres portadoras de endometriose com idade média de 32,52 anos. O estudo de Márki $G$ et al. (2017), observou dados similares no qual a idade média foi de 33,87 anos. Verificamos que $66,43 \%$ das participantes apresentaram impactos negativos na qualidade de vida em função da endometriose. Moradi $M$ et al. (2014), verificou que quase metade das mulheres tem a vida afetada pela endometriose, o que é similar ao observado neste estudo. Além disso, a endometriose é uma das principais causas de ônus econômico e qualidade de vida comprometida em uma porcentagem muito grande de mulheres asiáticas (DAI Y, et al., 2018).

Dor apresentou os escores de 10,7484 e 5,5875 para muitas vezes e sempre, respectivamente. A dor decorrente da endometriose na maioria dos casos afeta a vida social da mulher em seu período potencialmente ativo (NASCIMENTO R, et al., 2013). As mulheres com endometriose geralmente sofrem de dor intensa e outras consequências debilitantes que resultam em comprometimento da qualidade de vida (DAI 
Y, et al., 2018). Algumas mulheres experimentam dor apenas na menstruação. No entanto, outras descrevem sentir dor pélvica não relacionada à menstruação. Outros sintomas identificados como dolorosos por causa da endometriose são dor na bexiga, dor ao urinar, dor no intestino, dor durante e após a relação sexual e dor nas articulações na parte superior das pernas (JONES G, et al., 2004). Os sintomas de dor mais comumente atribuídos à endometriose são dismenorreia, dispareunia e dor pélvica crônica (dor pélvica não cíclica e não menstrual).

Aproximadamente 10 a $20 \%$ das mulheres em idade reprodutiva sofrem de endometriose, com 70 a $90 \%$ delas relatando dor pélvica crônica (DPC), dismenorreia, dispareunia, infertilidade e distúrbios menstruais (WINKEL CA, 2003).

Um fator relevante para a mulher é o advento da maternidade e a endometriose é um fator que impacta negativamente (Tabelas 5 e 6) sua qualidade de vida e o seu conceito de mulher uma vez que conflitos intrapsíquicos e turbulências interpessoais são observados em mulheres inférteis obliterando sua auto percepção sexual conflitando sexualidade e infertilidade, uma vez que pacientes acreditam que a gravidez faz parte da essência da mulher e assim, a possibilidade de dar à luz, pode colapsar (LEON I, 2010; VILA ACD, et al., 2010).

Além do relato da dor como fator de impacto negativo na qualidade de vida, sintomas como alterações de humor, depressão e irritabilidade estão presentes em mais de $60 \%$ das mulheres e $40 \%$ delas apresentam algum tipo de incapacidade na realização de suas atividades sociais. Dessa forma, a dor é responsável direta pela baixa qualidade de vida, perda de bem-estar físico, mental e social, associado a uma limitação no trabalho, estudo, vida social e sexual sendo obrigadas há inúmeras passagens por diferentes médicos frequentemente sem obtenção de sucesso nos tratamentos (NASCIMENTO R, et al., 2013).

Intensas modificações físicas e psicológicas estão ocorrendo na adolescência a qual é dividida em inicial (10 a 14 anos), média (14 a 17) e tardia (acima de 17 anos). Nesse contexto, ginecologista deve estar preparado em atender e orientar pacientes jovens em função de sua responsabilidade em direcionar a saúde dessas jovens mulheres quanto a sexologia, doenças infecciosas, fisiologia genital, sintomas entre outras patologias como a endometriose (GIORDANO MV e GIORDANO LA, 2009). Mulheres adultas afirmam a manifestação de sintomas pélvicos antes dos 20 anos, há uma proporção de $70 \%$ das adolescentes, com manifestações de dismenorreia e sangramentos irregulares (BROSENCE I e BENARGIANO G, 2011; SEPULCRI RP e AMARAL VF, 2007). Um drama na adolescência é a demora em diagnosticar a endometriose com tempo médio de 10 anos (ARRUDA MS, et al., 2010).

Verificamos que mulheres jovens entre 14-20 anos apresentaram maior escore no domínio Dor (escore $2,911, p=0,0342$ ). Adolescentes possuem lesões avermelhadas que são mais ativas produzindo maior quantidade de prostaglandinas que promovem contração intensa do músculo liso que incluem contrações do miométrio e intestinas (SEPULCRI RP e AMARAL VF, 2007). Assim, adolescentes com endometriose sentiriam mais dor do que pacientes adultas.

A endometriose interfere nas atividades profissionais apresentando relação direta entre dor crônica e sintomas psicológicos (estresse, ansiedade e depressão). Além disso, há a sobrecarga do cuidado familiar bem como do ambiente social e profissional sobre as mulheres, amplificando as dores e consequentemente o cansaço (BORGES CS, et al., 2009). Nesse contexto, verificamos que a endometriose interferiu nas atividades profissionais, pois mais de $29,21 \%$ reportaram serem afetadas, levando a um sentimento de culpa potencializando seus sintomas psicológicos.

Foi observada interferência da endometriose na relação mãe-filhos em 22,99\% das participantes com escore médio de 1,384, sendo o menor dentre as seções do questionário modular. Borges CS et al. (2009), verificaram não existir relevância entre as mulheres que possuem endometriose e a dificuldade em cuidar dos filhos, sendo essas informações compatíveis ao observado em nosso estudo.

Verificamos que $32,26 \%$ das mulheres relataram evitar relações sexuais por apresentarem dispareunia. Esse é o segundo sintoma mais prevalente, contribuindo para o diagnóstico patognomônico da endometriose com ocorrências de inflamações, aderências, sangramentos locais e consequentemente dor durante a 
estimulação das lesões no ato sexual (NOGUEIRA-SILVA C, et al., 2015; SANTOS TMVS, et al., 2012). A dispareunia está correlacionada à depressão e ansiedade em $90 \%$ das portadoras ocasionando redução da libido, autoestima, reduzindo frequência e interesse sexual origina forte sentimento de culpa (MATTA AZ e MULLER MC, 2006).

É frequente, por parte das mulheres, uma aversão da interpretação psicológica feita pelo médico sobre sua doença, pois ele foca em alterações anatômicas e funcionais, e não encontrando etiologia elas podem ser encaminhadas à psiquiatria, uma vez que são interpretadas como pacientes "psicossomáticas". Isso justifica os dados obtidos neste estudo, em que $35,47 \%$ das participantes referiram que sente em sua maioria um desconhecimento da classe médica em relação à doença (MATTA AZ e MULLER MC, 2006).

No tratamento da endometriose, é difundida a cirurgia e a terapia de supressão ovariana. Caso haja suspeita clínica de endometriose leve ou mínima apresentada com dor pélvica pode-se iniciar um tratamento empírico com anticoncepcionais orais. Se não houver melhora em três meses pode-se usar análogos de hormônio liberador de gonatrofina ( $\mathrm{GnRh}$ ), ou ainda, com suspeitas de aderências, indica-se a cirurgia (AOKI $\mathrm{T}, 2007)$.

O tratamento da endometriose está intrinsecamente relacionado aos sentimentos das pacientes. Aoki T (2007), verificou que $42,5 \%$ das participantes referiram consequências negativas do tratamento em relação à sua qualidade de vida, dentre elas o ganho excessivo de peso, diversos efeitos colaterais relacionados a medicações, estresse associado à antecipação da maternidade. O autor constatou que 10\% interromperam o tratamento médico relatando frustração. No atual estudo foi observado que $40,39 \%$ das mulheres sentiamse frustradas com o tratamento, e que as mulheres sempre apresentam dificuldades em lidar com os efeitos adversos do tratamento (VILA ACD, et al., 2010)

A dismenorreia e a infertilidade são sintomas que impactam diretamente a vida conjugal, social, profissional e na capacidade reprodutiva das mulheres, sendo a infertilidade o fator que afeta a grande maioria delas (64.8\%) com idade média de 34 anos com tempo médio de infertilidade de 4 anos (DE MARQUI ABT, 2014; MENGARDA CV, et al., 2019). Nesse contexto, das 640 participantes 84,14\% consideraram relevante a dificuldade de concepção, contudo das que responderam a seção $F$ (Gravidez) verificou-se que $55,34 \%$ das mulheres avaliadas são afetadas psicologicamente pela incapacidade de gerar filhos.

\section{CONSIDERAÇÕES FINAIS}

A Dor é um dos principais fatores causador da baixa qualidade de vida bem como o fato da impossibilidade de engravidar e ou manter a gravidez. Além disso, as mulheres se apresentaram descontentes com a classe médica em relação ao conhecimento sobre a doença bem como frustradas com os tratamentos. Assim, a Dor decorrente da endometriose afeta a vida social da mulher, altera seu interesse sexual, modifica sua concepção de mulher devido à infertilidade, ocasionando alterações de humor, depressão e irritabilidade com impacto negativo na qualidade de vida uma vez que a mulher não tem controle e se sente impotente diante da endometriose.

\section{AGRADECIMENTOS}

À Fundação de Amparo ao Ensino e Pesquisa da Universidade de Mogi das Cruzes (FAEP/UMC), à Coordenação de Aperfeiçoamento de Pessoal de Nível Superior (CAPES), à todas as mulheres que dedicaram seu precioso tempo em responder os questionários desta pesquisa.

\section{REFERÊNCIAS}

1. AOKI T. Avaliação da classificação histológica da endometriose observada em implantes de mulheres portadoras de endometriose pélvica superficial e profunda. Revista Brasileira Ginecologia Obstetricia 2007; 11(29): 568-574.

2. ARRUDA MS, et al. Endometriose profunda: aspectos ecográficos. Femina 2010; 38(7): 367-372.

3. BORGES CS, et al. Intervenção cognitivo-comportamental em estresse e dor crônica. Arquivos de Ciências da Saúde 2009; 16(4): 181-186. 
4. BROSENCE I, BENAGIANO G. Endometriosis, a modern syndrome. J Medical Research 2011; 133(6): 581-593.

5. BUCK LOUIS GM, et al. Incidence of endometriosis by study population and diagnostic method: the endo study. Fertil Steril 2011; 2(96): 360-365.

6. CARDOSO EPS, et al. Endometriose em diferentes faixas etárias: perspectivas atuais no diagnóstico e tratamento da doença. Ciências e Práxis 2011; 8(4): 53-58.

7. DAI Y, et al. A review of the risk factors, genetics and treatment of endometriosis in Chinese women: a comparative update. Reproductive Health 2018; 15(1: 82): 1-12.

8. DE MARQUI ABT. Endometriose: do diagnóstico ao tratamento. Revista de Enfermagem em Atenção Saúde Online 2014; 2(3): 97-105.

9. DONATTI L, et al. Patients with endometriosis using positive coping strategies have less depression, stress and pelvic pain. Einstein 2017; 1(15): 65-70.

10. GIORDANO MV, GIORDANO LA. Contracepção na adolescência. Ver Adolescência \& Saúde 2009; 6(4): 11-16.

11. HERNÁNDEZ-VALENCIA M. Diagnóstico de la endometriosis. Perinatol Reprod Hum 2009; (23):25-29.

12. JONES G, et al. The impact of endometriosis upon quality of life: a qualitative analysis. Journal of Psychosomatic Obstetrics \& Gynecology 2004; 25(2): 123-130

13. LEON I. Understanding and treating infertility: Psychoanalytic considerations. J American Academy of Psychoanalysis and Dynamic Psychiatry 2010; 38(1): 47-75.

14. LUIZ RP, et al. Incidencia de la endometriosis en mujeres con infertilidad: características clínicas y laparoscópicas. Ginecologia y Obstetricia de Mexico 2005; 73: 471-476.

15. MÁRKI G, et al. Physical pain and emotion regulation as the main predictive factors of health-related quality of life in women living with endometriosis. Human Reproduction 2017; 32(7): 1432-1438.

16. MATTA AZ, MULLER MC. Uma análise qualitativa da convivência da mulher com sua endometriose. Psicologia, saúde \& doenças 2006; 1(7): 57-72.

17. MENGARDA CV, et al. Validação de versão para o português de questionário sobre qualidade de vida para mulher com endometriose (Endometriosis Health Profile Questionnaire-EHP-30). Revista Brasileira de Genecologia \& Obstetrícia 2019; 30(8): 384-392.

18. MINSON FP, et al. Importância da avaliação da qualidade de vida em pacientes com endometriose. Rev Bras Med Ginecol Obstet 2012; 1(34): 11-15.

19. MORADI M, et al. Impact of endometriosis on women's lives: a qualitative study. BMC Womens Health 2014; 14(123): $1-12$.

20. NACUL AP, SPRITZER PM. Aspectos atuais do diagnóstico e tratamento da endometriose. Revista Brasileira de Ginecologia \& Obstetrícia 2010; 32(6): 298-307.

21. NASCIMENTO R, et al. Avaliação da qualidade de vida e controle da dor em pacientes portadores de endometriose após inserção do sistema intrauterino liberador de levonorgestrel (SIU-LNg). Arquivos catarinenses de medicina 2013; 42(4): 21-27.

22. NOGUEIRA-SILVA C, et al. Validation of the Portuguese Version of EHP-30 (The Endometriosis Health Profile-30). Acta Médica Portuguesa 2015; 28(3): 347-356.

23. SANTOS TMVS, et al. Tempo transcorrido entre o início dos sintomas e o diagnóstico de endometriose. Einstein 2012; 1(10): 39-43.

24. SEPULCRI RP, AMARAL VF. Endometriose pélvica em adolescentes: novas perspectivas. Femina 2007; 35(6): 355362.

25. SOLIMAN AM, et al. The direct and indirect costs associated with endometriosis: a systematic literature review. Human Reproduction 2016; 4(31): 712-722.

26. VILA ACD, et al. A vivência de infertilidade e endometriose: pontos de atenção para profissionais de saúde. Psicologia, Saúde \& Doenças 2010; 11(2): 219-228.

27. WINKEL CA. Evaluation and management of women with endometriosis. Obstetrics \& Gynecology 2003; $102(2)$ : 397408. 